\title{
Zwei permische Ableitungssuffixe
}

\section{Das syrjänische Nomensuffix -na}

Im Syrjänischen gibt es ein selten vorkommendes denominales Nomensuffix -na. Bei Wiedemann (Gr. 55) finden wir vier diesbezügliche Beispiele: (Wied.) P. gozna 'zwei Balken unter dem Fussboden'; (WıEd.) gyžna 'Krätze, juckender, krätzartiger Ausschlag, Scropheln'; (Wiснм.--Uот.) S. Lu. Le. P. gižna, I. gižna 'Hautausschlag (S. Lu. Le. P. I.), Schorf (auf einem Ausschlag) (Lu. Le. P. I.); Skrofeln (S. Lu. Le. P. I.)', weitergebildet: S. gižnaeśšini 'sich mit Ausschlag bedecken (Haut)'; (Wied.) P. šoina, soinan 'Grab'; (WiEd.) vyina 'Gränze, Mass'. - Dasselbe Suffix - na ist auch noch in den folgenden Wörtern vorhanden: (WICHм.-Uoт.) S. pidna- 'Tiefe'. Vermutlich dürfte auch das Element - na folgender beider Wörter mit diesem Nomensuffix -na identisch sein: (WICHм.-Uot.) S. ilna: meśán sịl vit kịmin îlna 'ungefähr fünf Klafter von mir entfernt'; (Fokos-Fucris SyrjWb.) Le. vilna: muina vilna! 'wie hoch'; Le. muina vilna vel-doras pu-jilin! 'wie hoch ist er oben auf dem Baum!'

Wiedemand (Gr. 55) behandelt das syrj. Suffix -na im Zusammenhang mit dem Suffixelement -ria (z.B. gidria 'Stall', vgl. gid 'Stall, Hof, Hürde'), woraus wir darauf schliessen können, dass er die beiden Suffixe für etymologisch gleichen Ursprungs hält. Die Verknüpfung der Suffixe - na und -na ist phonetisch darum nicht möglich, da das syrj. $n$ dem $\dot{n}$ keinesfalls entsprechen kann.

Lehtisalo (MSFOu. 72 S. 120) bringt das syrj. Suffix -na mit dem finnisch-ugrischen denominalen Nomensuffix ${ }^{*} n$ in Zusammenhang. Er nimmt an, dies -na dürfte eventuell ein zusammengesetztes Suffix sein. 
Die Endung -na in den bisher aufgezählten Wörtern ist tatsächlich ein zusammengesetztes Suffix. Meiner Ansicht nach entstand das Nomensuffix -na aus der Verknüpfung zweier Suffixe, des inessivischen -in, beziehungsweise des Instrumentalsuffixes - $m$ mit dem Adjektivsuffix $-a$, das die Bedeutung 'mit irgendeiner Sache versehen' hat.' Die Wörter mit dem Suffixe -na waren ursprünglich Attribute (ihrer Wortart nach Adjektive), neben denen als determiniertes Wort irgendein Hauptwort stand. Später nahmen dann diese Attribute mit der Endung -na die Bedeutung der darauffolgenden determinierten Wörter in sich auf und wurden selber zu Hauptwörtern. Das ist semasiologisch genommen durchaus möglich. Es kommt nämılich recht häufig vor, dass dem einen Bestandteil gewisser fester attributivischer Konstruktionen die Bedeutung des zweiten Bestandteiles beigefügt wird (Goмвосz ÖM. I S. 25).

Im Sinne des Obenangeführten dürfte die Entwicklung der obigen auf -na endenden Hauptwörter auf folgende Weise vonstatten gegangen sein (im Zusammenhange der einzelnen Wörter erwähne ich die rekonstruierte Attributivkonstruktion, in der [selbst oder in ihr ähnlichen Konstruktionen] das betreffende Hauptwort zustande kommen konnte).

Die Bedeutung des Wortes (Wied.) P. gozna 'zwei Balken unter dem Fussboden' (Grundwort: goz 'Paar') <*gozena ker dürfte 'Balken mit seinem Paar, paarweise [vorhandener] Balken' gewesen sein. Die ursprüngliche Bedeutung des Wortes (WIED.) gyžna 'Krätze', (WICHм.--Uoт.) gižna 'Hautausschlag usw.' (Grundwort: giž 'Nagel, Klaue, Kralle') < *gižna gizjalem war vermutlich 'Kratzen mit den Nägeln, Krallen'. Ebenso: (WIED.) P. šoina 'Grab' (Grundwort: šoi 'Leiche, Aas') < šojena in 'Ort mit einem Leichnam, mit einem Leichnam [versehener] Ort'. In dem auslautenden $-n$ der Wortgestalt (WIED.) šoinan

1 Der Zusammenhang zwischen dem Instrumental- und Adjektivsuffix - $a$ ist im folgenden Worte auch heute noch klar: (Wicн м. - Uor.) I. podena 'Fussgänger', P. podena-mort id.; (Fokоs-Fcchs SyrjWb.) V. podena 'zu Fuss gehend (Adj.)'; vgl. poden 'zu Fuss'. Das Grundwort: (Wrснм. - Lот.) S. pod 'Schlittenfessel' $\sim$ votj. (Wıснм.) G. pîd, M. J. MU. pid 'Fuss' (vgl. Wichmasi WotjChrest. ${ }^{2}$ 93; Uotila Syrj Chrest. 137). 
'Grab' können wir aller Wahrscheinlichkeit nach das syrj. Wort (Fokos-Fuchs SyrjWb.) in 'Ort, Stelle' auffinden: * šoina in > šoinnan (WIED. šoinan). -- In den bisher besprochenen Wörtern ist das erste Element des Suffixes -nn --- wie aus dem bisher Angeführten hervorging -- mit dem Instrumentalsuffix identisch. In den folgenden Beispielen ist das erste Element des Suffixes -na wohl ein Inessivsuffix: (Wrev.) vyina 'Gränze, Mass' (Grundwort: viin: vijen 'bis', rgl. Wichm.---Uorr.) < *ijina in 'am Ende [befindlicher] Ort'; (Wicнм.-Uот.) S. pidna- 'Tiefe' (Grundwort: V. pid 'Tiefe', Fokos-Fuchs SyrjWb.) < * pidina in 'in der 'Tiefe [liegender] Ort'. Auf Grund der angeführten Beispiele können wir bei dem Wort ilna auf die Bedeutung 'Weite', bei dem Wort vilna aber auf dic Bedeutung. 'Höhe' folgern. Sie dürften aus Attributivkonstruktionen wie *ilina in $(>*$ *ilna in $>$ ilna) 'in der Ferne [befindlicher] Ort' (vgl. ilin 'weit'); *vilina in ( $>$ *vilna in > vilna) 'in der Höhe [befindlicher] Ort' (vgl. vilin 'oben'). In den erwähnten Beispielen werden die Formen ilna und vilna als insuffigierte Adverbien gebraucht.

Es ist durchaus nicht ungewöhnlich, dass das Substantivsuffix -na aus der Vorknüpfung des Inessiv- beziehungsweise Instrumentalsuffixes + Adjektivsuffixes zustande kam. Diesem Suffix ähnelt seiner Konstruktion nach das syrj. Adjektivsuffix -śa: lị̂-cośa 'zweijährig', eniśa 'jetzig, gegenwärtig'. Dies entstand nämlich aus der Zusammensetzung des Elativsuffixes is und des Adjektivsuffixes - $a$ (Fuchs KSz. 13 S. 98, FUF 30 S. 197, NyK 60 S. 314).

Für die Verbindung von Kasus- und Bildungssuffixen kömmen auch aus anderen finnisch-ugrischen Sprachen Beispiele angeführt werden. Das ungarische nomen possessi -Suffix $-i$ kann auch Adverbien (mostani 'jetzig', egyuttali 'zugleich vorkommend'), Hauptwörtern mit Kasussuffixen (helybeni 'örtlich') und Postpositionen (föld alatti 'unterirdisch', vég nélküli 'unendlich') beigefügt werden (Simony TMNy. I S. 597; Szinnyei $\mathrm{NyH}^{7}$ S. 100; D. Bıвтни Szóképz. S. 121). Das fimnische Adjektivsuffix -llinen kam gleichfalls aus der Verknüpfung von Kasusund Bildungssuffix (Adessivsuffix -lla, $-l l a ̈+$ Adjektivsuffix -inen) zustande: aamullinen 'morgendlich', onnellinen 'glück- 
lich, glückhaft, glückselig', kourallinen 'eine Handvoll'. Die ursprüngliche lokale Bedeutung des Suffixes -llinen ist in den folgenden Beispielen auch jetzt noch klar: maallinen 'irdisch. weltlich', taivaallinen 'himmlisch' (HAKuLINEN SKRK I S. $140-142$ ).

2. Das wotjakische Kollektivzahlwortsuffix -na

Es ist bekaunt, dass in den meisten finnisch-ugrischen Sprachen die Numeraladverbien mit Lokativ- oder Instrumentalsuffixen gebildet werden. Zum Beispiel ung. ketten 'zu zweit', hárman 'zu dritt', négyen 'zu viert'; wog. yūrman 'zu dritt, zu dreien', nillän 'zu viert, zu vieren'; syrj. kiken 'zu zweien', kujimen 'zu dreien'; tscher. KH. koktâ'n, U. ko kttân 'zu zweien'; finnisch kahden 'zu zweien', kolmen 'zu dreien'.

Den syrjänischen Zahlwörtern kiken 'zu zweien', kujimenn 'zu dreien' usw. können auch Possessivsuffixe beigefügt werden: kiknanim, kiknanid, kiknanis oder kikna.nis 'wir, ihr, sie zwei od. beide' (WIснм.-Uот.); V.S. nolna nis 'sie alle vier' (ebenda). Ebenso: etnam, eknam '(ich) allein', etnad, elnad '(du) allein', etnas, eknas '(er) allein', etnanim, eknanim '(wir) allein', etnanid, eknanid '(ihr) allein', etnanis, eknanis '(sie) allein'. In diesen Zahlwörtern ist das Instrumentalsuffix - en vor Possessivendungen $(-m,-d,-s,-n i m,-n i d,-n i s)$ in der ursprünglicheren Lautform $-n a$ vorhanden.

Aus den syrjänischen Zahlwörtern wie z.B. kiknanĭm 'wir zwei od. beide' entstanden durch Abstraktion die Kollektivzahlwörter V. kiknan, P. kikna •n, Ud. kiklan 'beide' (Wrснм.Uот.); Ud. V. S. Le. Pr. Vm. kujimnan 'alle drei (attributiv)' (Fokos-Fuchs SyrjWb.); V. VO. nolnan 'alle vier (attributiv)' (ebda). An die auf diese Weise zustande gekommenen Zahlwörter kiknan, kujimnan wurden dann wieder die Possessiv-

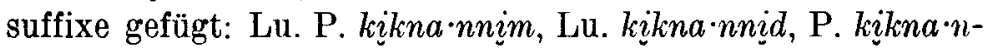
nit, Lu. P. kikna·nnis 'wir, ihr, sie beide' (WІснм.-Uот.), V. Lu. kujimna·nnis 'alle drei, zu dreien' (ebda). Die Formen Ud. kiklan 'beide' (W гснм.--Uот.), Ud. kujimla.nnis 'alle drei, zu dreien' (ebda) kamen durch die Dissimilation $n>l$ aus 
den Formen kiknan, kujimnan zustande (vgl. Wiedemans Gr. 154-155; Uotila FUF 29 S. 23).

Wiedemann (Gr. 154-155) und Aminoff (JSFOu. 14:2 S. 39) identifizieren mit dem in den syrjänischen Zahlwörtern etnam, eknam 'ich allein' usw., kiknanim 'wir zwei od. beide' auffindbaren Instrumentalsuffix $n a$ das Element $n a$ der wotj. Zahlwörter ognam 'ich allein', ognad 'du allein', ognaz 'er allein', ognamy 'wir allein', ognady 'ihr allein', ognazy 'sie allein', lyknamy 'wir beide', kyknady 'ihr beide', kyknazy 'sie beide'. Im Syrjänischen kommt das Instrumentalsuffix -en vor Possessivsuffixen tatsächlich in der Lautform na zum Vorschein (zum Beispiel: velen 'mit dem Pferd', velnam 'mit meinem Pferd'), im Wotjakischen dagegen lautet sowohl bei der absoluten als possessiven Deklination das Instrumentalsuffix -en, - $\hat{\imath} n$, -in: B. vuzaz suurs maneten 'er verkaufte es für 1000 Rubel'; G. semiàien?̂m, J. śemiåienim 'mit meiner Familie' (WICHManN WotjChrest. $^{2}$ 139, 143). Das Element na der wotjakischen Zahlwörter ognam, kyknamy usw. ist also nicht mit dem Element na der behandelten syrjänischen Zahlwörter in Zusammenhang zu bringen.

Munkícsi (Budenz-Alb. S. 289-291) hat das Element na der Wortformen syrj. kiknanim 'wir zwei od. beide'; wotj. kiknami 'wir beide' usw. mit dem Suffixe -rièk der tscher. Kollektivzahlwörter ko·ranèk 'alle beide', nalınièk 'alle vier' verglichen. Wie aus dem bisher Angeführten hervorging, ist das na der Zahlwörter des syrj. kiknanim usw. mit dem Instrumentalsuffix identisch. Das in der wotjakischen Form kiknami 'wir beide' vorhandene na kann aber schon darum nicht dem tscheremissischen Suffixe -rièk entsprechen, weil das tscheremissische Kollektivzahlwort laut BeKe (Cseremisz nyelvtan 280) aus der Zusammensetzung des Lokativsuffixes $-n$ und der verstärkenden Partikel -ok entstanden ist. Budenz (Ugor Alaktan 321) und Foxos (NyK 36 S. 421-424) halten das Element -na ebenfalls für ein Kollektivzahlwortsuffix.

In der heutigen wotjakischen Sprache werden die Numeraladverbien mit der Bedeutung 'zu zweit, zu dritt' usw. mit Hilfe des Adverbs čošän 'zusammen, nebeneinander' oder der Postposition kuźa 'längs, entlang' ausgedrückt. (MUNK.): K. ke̊k 
rošän 'je zu zwei’, K. liün cos̆än 'zu dreien'; вить куyзя 'впятером. ${ }^{1}$

Wahrscheinlich waren - ähnlich wie in den anderen finnischugrischen Sprachen - auch im Wotjakischen die mit dem Instrumentalsuffix gebildeten Numeraladverbien gebräuchlich. Darauf weisen folgende mit dem Instrumentalsuffix geprägte Zahlwörter wie (Musk.) s. ogän, odigän 1. 'einzeln, einzelnweise; 2. 'allein, alleinstehend'; s. ogän-oyän 'einzeln'; (Wiso.) kyjlen zu zwei, paarweise; (WICнм.) MU. ogen kiken 'einer nach dem anderen' hin.

Meiner Ansicht nach ist das $n$ in dem Suffix -na der wotjakischen Kollektirzahlwörter (Musk.) s. ognam 'ich allein', ognad 'du allein' usw., (Wred.) kykna 'beide, ihrer zwei', (Munk.) S. lįknami 'unser zwei, wir beide', killnadi 'ihr zwei', kiknazi 'sie zwei', S. kuinnaze̊, K. künnaze 'ihrer drei', S. nilnazi 'ihrer vier, alle viere' usw., mit dem Instrumentalsuffix identisch, das Element - $a$ dagegen ein Adjektivsuffix. Das Suffix - $a$ - indem es sich den Numeraladverbien ogän 'einzeln, allein', *kiken 'zu zweien' usw. anschloss - adjektivisierte diese und bildete so aus ihnen Kollektivzahllwörter: ogän 'allein': *ogäna $>{ }^{*}$ ogna 'alleinstehend', vgl. (*ogänam >) ognam 'ich allein'; *kiken 'zu zweien': *kiliena > kikina 'beide'; *kikenami > kịknami 'wir beide'. - Der Ausfall der Laute $\ddot{a}, e$ in der zweiten Silbe der Formen ${ }^{*}$ ogänam $>$ ognam, *kikena $>$ kilina usw. ist regelmässig. Es fällt nämlich in den permischen Sprachen, falls sich dem konsonantisch auslautenden Worte irgendein Suffix anschliesst, der Vokal der vor dem Suffix stehenden Silbe häufig weg z.B.: (Мusк.) K. pules, G. polis 'Ruder': S. pulsal-, G. polsal- 'rudern', (Muxk.) S. puläs 'Riss, Spalte (z.B. an der Haut)': S. pulsal- 'auftrennen, lostrennen, abschälen, abziehen (z.B. die Haut vom Fleische)'; (Munk.) S. J. M. G. kužim, K. kužem 'Kraft, Stärke, Macht': S. kužmo 'stark, kräftig, mächtig' (vgl. Wiedemaxx Gr. 35).

Wie bekannt, blieb das permische Adjektivsuffix ${ }^{*}-a$ im

1 Русско-удмуртский словарь. Москва 1956. Im Anhang des Wörterbuches: Перевочиков Краткий очерк грамматики удмуртского нзыка S. 1313. 
Syrjänischen als - $a$ erhalten; im Wotjakischen dagegen wurde es nach einem $o$ oder $u$ in der Stammsilbe zu einem $o$. Später wurde dieses Adjektivsuffix -o auch bei solchen Wörtern allgemein, in deren Stammsilbe kein o oder $u$, sondern irgendein anderer Vokal vorhanden war. Die ursprüngliche Form -a dieses Suffixes kommt heute nur mehr selten, lediglich in einzelnen Ausdrücken vor, wie z.B. (Aмrs.) keñyra-šıd 'ryynisoppa' (vgl. Aunvoff JSFOu; 14:2 S. 22; Lakó Fgr. Ért. II S. 32; Radanovics NyK 61 S. 81). - Ebenso blieb das Adjektivsuffix in der Lautform $a$ in dem wotjakischen Kollektivzahlwortsuffix na erhalten. Das $a$ in dem Kollektivzahlwortsuffix $n a$ wurde vermutlich deswegen nicht in ein o verwandelt, da sein Zusammenhang mit dem Adjektivsuffix $a$ für das Sprachgefühl bereits verblich.

Zur Unterstützung des Obenangeführten erwähne ich einige Fälle, bei denen im Wotjakischen kein Lautwandel $a>0$ gcschah.

Das Lativsuffix $a$ ist im allgemeinen im Wotjakischen in der Lautform a vorhanden: (Munk.) S. kema 'lange, lange Zeit', (Munk.) S. kuźa, K. küźa 'längs, entlang'. Dann und wann kommt jedoch nebst einem $a$ auch das Lativsuffix in der Lautform o vor: (Wiснм.) MU. aźla 'vorher, zuerst' $\sim$ (Munk.)

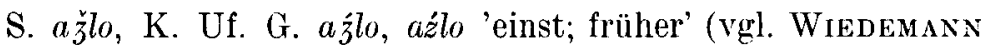
Gr. 47, 54; Uotila MSFOu. 65 S. 130, 198-200).

Auch bei dem wotjakischen Adjektivsuffix -ta, $-d a$ ging kein Lautwandel $a>o$ vor sich: (Munk.) S. minda, K. me̊nda 'eine gewisse Quantität habend', (W Iснм.) (r. mînda, J. minda 'so viel wie'; (Musk.) S. pasta 'eine Breite habend, so breit wie...' (vgl. Wiedemann Gr. 47; Beke AIJH 2 S. 339-348).

Es sei noch erwähnt, dass das Kollektivzahlwortsuffix na auch im folgenden Wort auffindbar ist: (WIEv.) votśna, vitśna 'alle zusammen' (vgl. vols', vits' 'alle, jeder, ganz' ebda); (Munk.) S. vočnami, G. vićnami 'wir alle', S. vočnadi, G. vićnadi 'ihr alle' (vgl. Wiedemann Gr. 47; Munkácsi Budenz-Alb. S. 289-291; Fokos NyK 36 S. 422).

Es bedürfen noch folgende wotjakische Formen einer Erklärung: (W гснм.) G. ognị̂n 'allein'; ogriam 'ich allein', ogñad 'du allein', ognaz 'er allein'; (Munk.) G. ograde 'ihr allein'. Das 
Element $-\hat{n} \hat{\imath} n$, beziehungsweise -ria- in diesen kann wegen der Verschiedenheit der Konsonanten nicht mit dem jetzt behandelten wotjakischen Kollektivzahlwortsuffix -na identisch sein. Es ist beachtenswert, dass diese Formen nur in der (i. Mundart rorkommen. In derselben Mundart (wie auch in B. Dialekt) gibt es ein sekundäres, aus der Postposition din??̂n 'bei' entstandenes Adessirsuffix -rị̣̂n, zum Beispiel: G. mumezṇ̃̂n 'bei ihrer Mutter', B. mońnam 'bei mir' (vgl. Wichmaxs WotjChrest." 135). Nun ist die Erklärung von Fokos ( $\mathrm{XyK} 36 \mathrm{~S}$. 247) am wahrscheinlichsten, derzufolge dasselbe Adessirsuffix auch in den wotjakischen Formen G. ognị̂n 'allein', ognam 'ich allein' usw. vorhanden ist (deren Grundwort og 'ein' ist).

KáROLY R.ADANOVICS 